\title{
Hospitalist and Intensivist Experiences of the "Open" Intensive Care Unit Environment: a Qualitative Exploration
}

\author{
Lekshmi Santhosh, M.D., M.A.Ed. ${ }^{1,2}$ and Justin Sewell, M.D., Ph.D., M.P.H. ${ }^{3}$ \\ 'Division of Pulmonary/Critical Care Medicine, University of California-San Francisco, S1 183, 505 Parnassus Avenue, San Francisco, CA, USA; \\ ${ }^{2}$ Division of Hospital Medicine, University of California-San Francisco, S1 183, 505 Parnassus Avenue, San Francisco, CA, USA; ${ }^{3}$ Division of \\ Gastroenterology, Zuckerberg San Francisco General Hospital, University of California-San Francisco, San Francisco, CA, USA.
}

\begin{abstract}
BACKGROUND: Most U.S. academic medical centers employ "closed" intensive care units (ICUs), where critically ill patients are admitted under the supervision of intensivists managing dedicated ICU teams. Some centers utilize a unique "open" ICU structure, where primary services longitudinally follow patients who become critically ill into the ICU with intensivist comanagement. The impact of open ICUs on patient care and education of trainees has not been well-characterized.
\end{abstract}

OBJECTIVE: The objective of this study is to characterize affordances and barriers to education and patient care, from the perspectives of hospitalists and intensivists teaching in the ICU.

DESIGN: We conducted semi-structured interviews with hospitalist and intensivist faculty at a large academic medical center with an open ICU structure. We coded deidentified interview transcripts to inductively analyze the data for themes and subthemes.

PARTICIPANTS: We recruited hospitalist and intensivist faculty members who attend on teaching services in the open ICU system.

APPROACH: Given the complexity of multiple teachers and learners in the ICU environment, we selected shared mental models as our primary theoretical lens through which we analyzed and interpreted our data.

KEY RESULTS: We identified three main themes regarding education in the open ICU system: (1) communication challenges, (2) educational barriers and affordances, and (3) structural barriers and affordances. Hospitalists and intensivists agreed on some barriers and facilitators to education, such as continuity of care, yet they disagreed on others. Specifically, hospitalists and intensivists had a shared mental model regarding barriers to patient care and education in the open ICU structure, but had divergent opinions regarding the affordances of the structure, such as continuity and availability of ICU expertise.

CONCLUSIONS: The open ICU environment presents facilitators and barriers to trainee education and patient care. Our findings can be leveraged to improve communication, education, and patient care on both hospitalist and ICU teams.

Electronic supplementary material The online version of this article (https://doi.org/10.1007/s11606-020-05835-w) contains supplementary material which is available to authorized users.

Received September 11, 2019

Accepted April 1, 2020

Published online May 27, 2020
KEY WORDS: comanagement; interdisciplinary; communication; ICU; consultation.

J Gen Intern Med 35(8):2338-46

DOI: $10.1007 / \mathrm{s} 11606-020-05835-w$

(C) Society of General Internal Medicine 2020

\section{INTRODUCTION}

Intensive care units (ICUs) are specialized units in the hospital with higher medical complexity, urgency of decision-making, and time pressures of medical care, thus making them demanding sites both for professional work and for learning. Most academic medical centers utilize "closed" ICUs. In such units, critically ill patients are admitted under the supervision of intensivists managing dedicated ICU teams; when patients transfer into closed ICUs, either from the medical wards or from the emergency department, the preceding admitting team (e.g., medical or surgical team) ceases to be involved in the patient's care. This presents breaks in continuity for patients and providers alike. However, some academic centers and many community-based medical centers utilize an "open" ICU structure, ${ }^{1}$ where primary services longitudinally follow patients into the ICU from the emergency room or the ward who become critically ill and the intensivist team comanages aspects of patients' care. A national survey showed that $68 \%$ of hospitalists reported caring for ICU patients, suggesting this structure is common. ${ }^{2}$ Hospitals that are not affiliated with academic medical centers are more likely to have open ICUs; one study estimated that $41 \%$ of community programs had open ICU models. ${ }^{3}$ Hospitals that are more rural or hospitals lacking resources to hire dedicated intensivists also may be more likely to have open ICU models.

In open and closed ICUs alike, the medical complexity, time pressures, diverse levels and professions of learners, and difficulties communicating with patients and families at the end-of-life contribute significant challenges to education and patient care. ${ }^{4}$ These challenges impact the entire continuum of medical learners, from medical students to ICU fellows. However, limited literature has addressed education in this complex setting, ${ }^{5,6}$ and the perspectives of practicing hospitalist and ICU faculty are not well understood, especially in the academic open ICU model. There are two teams of physicians-hospitalists and intensivists - who practice medicine and teach trainees in 
the same location and with the same patients and families, yet who may have very different perspectives on education, patient care, and preferred organizational structure for the ICU. Elucidating faculty perspectives on educational challenges in the ICU from both groups could help promote improved education and even quality of care. Moreover, affordances and barriers of the open ICU system have not previously been well-characterized, so this study could serve as an initial exploration into the broader questions about ICU structure and education.

The objective of this study was to characterize perceived affordances and barriers to both education and patient care, from the perspective of hospitalists and intensivists, in the context of the open ICU structure. We designed the study to address three primary research questions:

1. What affordances and barriers to trainee education are present in the open ICU system?

2. What affordances and barriers to patient care are present in the open ICU system?

3. How do perceptions of hospitalists and intensivists teaching in the ICU differ?

\section{METHODS}

\section{Study Design}

We conducted semi-structured interviews of hospitalist and intensivist faculty at the University of California-San Francisco (UCSF), a large academic medical center with an open ICU structure. Primary interview questions (Box 1) were developed through iterative discussions between authors. We recruited teachers (and not learners) to enrich both content and pedagogical knowledge. Participants completed 40-60 min semi-structured one-on-one in-person interviews with LS (who is a female board-certified pulmonologist and intensivist and who has Masters-level training in education research) in an office at the hospital. LS was familiar with and had professional working relationships with all participants; she explained her rationale for performing the study with each participant. Interviews were audio recorded, deidentified, and transcribed verbatim; participants were not asked to review transcripts. Purposive sampling was employed to recruit hospitalist and intensivist faculty who attend frequently on the teaching service across the spectrum of experience and including both men and women. We planned to continue collecting interviews until we achieved information sufficiency. ${ }^{7}$ Clinical service chiefs sent recruitment emails to all teaching-service attending faculty encouraging participation in the study. Study participants opted in by replying to the email. The institutional review board of UCSF deemed the study exempt; all study participants indicated informed consent and participation in the study was voluntary.

\section{Box 1 Primary interview questions}

1. Please think about a time when a patient transitioned from the ICU to the ward and you thought the handoff experience was particularly challenging for the team. These challenges could relate to any portion of the procedure, including before the handoff, during the handoff, or after the handoff. Can you tell me about that encounter?

2. What do you see as the most significant challenges that

hospitalist/intensivist attendings experience while teaching in the ICU? What techniques or approaches do you use to address the challenges? 3. What do see you as the most significant challenges that learners experience while taking care of patients in the ICU? What techniques or approaches do you use to address these challenges?

4. Are there curricular or systemic factors that affect your ICU trainees in a positive or negative manner? Specifically, how does the open ICU affect training?

\section{Reflexivity}

LS is an intensivist and hospitalist, and JLS is a gastroenterologist. Both authors have extensive workplace teaching experience, as well as formal training in Health Professions Education research, including qualitative methods (LS has a Master's degree in Health Professions Education and JLS a $\mathrm{PhD}$ in Health Professions Education).

\section{Conceptual Framework}

Given the complexity of multiple physician teachers and learners in the ICU environment, we selected the theoretical framework of shared mental models as a pertinent lens to analyze education in the open ICU environment. Shared mental models explain how individuals in large teams develop a shared understanding of the roles of team members and structures of work. ${ }^{8}$ Shared mental models have been associated with better mutual understanding between team members and are frequently studied in situations of transitions of care, so applying this framework to the open ICU would be instructive.

\section{Data Analysis}

We deidentified interview recordings and then coded the transcripts using Dedoose (Manhattan Beach, CA). We applied template analysis ${ }^{9}$ (a form of thematic analysis) to inductively develop codes and analyze the qualitative data. LS developed the initial coding template, which both authors discussed and modified; authors met repeatedly to reconcile and discuss differences in coding. The coding template was iteratively revised throughout the coding process. All 20 transcripts were coded independently by both LS and JLS and then reviewed together. All disagreements in coded sections of text were reconciled by consensus between the two coders, which results in a single, agreed upon code for each relevant section of text. We characterized barriers and affordances of the open ICU system related to education and patient care. For data analysis, excerpts of relevant quotations were selected to highlight themes and subthemes (Appendix). Member checking was not employed. 


\section{Current Team Structure and Processes}

The ICUs at UCSF are roughly cohorted into medical, surgical, cardiac, and neurological ICUs, but there is some overflow. Each ICU has 16 beds and each utilizes the open ICU model. Hospital medicine teams are comprised of one Medicine attending (typically a hospitalist), one senior resident physician, two intern physicians, and up to three medical students. The ICU teams are comprised of one ICU attending (typically a pulmonary/critical care physician or an anesthesia/ critical care physician), one ICU fellow, and four junior residents from internal medicine, emergency medicine, and anesthesia. Formally structured interactions do not exist between the ICU and Medicine teams, though attendings and teams are informally encouraged to check-in with each other daily. Both teams round separately at the bedside, and both attendings independently teach their respective teams.

\section{RESULTS}

Table 1 describes the 20 participants -12 hospitalists and 8 intensivists, all of whom worked in the open ICU model. After these 20 interviews, we achieved information sufficiency. We identified three main themes regarding education and patient care in the open ICU: (1) communication barriers and affordances, (2) educational barriers and affordances for trainees, and (3) structural barriers and affordances of the open ICU. Box 2 provides a summary of themes and subthemes.

\section{Box 2 Summary of themes and subthemes}

\begin{tabular}{|c|c|c|}
\hline $\begin{array}{l}\text { Communication } \\
\text { barriers and } \\
\text { affordances }\end{array}$ & $\begin{array}{l}\text { Educational barriers } \\
\text { and affordances }\end{array}$ & $\begin{array}{l}\text { Structural } \\
\text { barriers and } \\
\text { affordances }\end{array}$ \\
\hline $\begin{array}{l}\text { Within team } \\
\text { Intra-team } \\
\text { Interprofessional } \\
\text { Between teams } \\
\text { ICU—primary } \\
\text { Consultants } \\
\text { Transitions of } \\
\text { care/handoffs } \\
\text { To patients/families }\end{array}$ & $\begin{array}{l}\text { Competing demands } \\
\text { Educator demands } \\
\text { Learner demands } \\
\text { Service-education } \\
\text { balance } \\
\text { Time pressure } \\
\text { Distractions } \\
\text { Institutional support of } \\
\text { educators } \\
\text { Medical \& educational } \\
\text { complexity } \\
\text { Medical complexity } \\
\text { Diagnostic uncertainty } \\
\text { Procedural complexity } \\
\text { Team complexity }\end{array}$ & $\begin{array}{l}\text { Continuity vs. } \\
\text { fragmentation } \\
\text { Continuity } \\
\text { Fragmentation } \\
\text { ICU/non-ICU } \\
\text { balance } \\
\text { Role ambiguity \& } \\
\text { anxiety } \\
\text { Cognitive } \\
\text { disengagement } \\
\text { Delocalization } \\
\text { Expertise vs. } \\
\text { comanagement } \\
\text { Lack of ICU } \\
\text { expertise } \\
\text { Lack of ICU } \\
\text { curriculum } \\
\text { Job satisfaction } \\
\text { Scope of practice } \\
\text { Spaced learning }\end{array}$ \\
\hline
\end{tabular}

\section{Communication Barriers and Affordances}

Participants discussed how a variety of communication challenges in the open ICU system impacted patient care, including challenges communicating within the larger interprofessional team, challenges communicating between teams, challenges communicating with consultants, and challenges
Table 1 Characteristics of Participants

\begin{tabular}{|c|c|c|}
\hline \multirow[t]{2}{*}{ Characteristic } & Descriptive statistic & Characteristic \\
\hline & $\begin{array}{l}\text { Hospitalists, } \\
n(\%)\end{array}$ & $\begin{array}{l}\text { Intensivists, } \\
n(\%)\end{array}$ \\
\hline Total number & $12(60)$ & $8(40)$ \\
\hline Female gender, no. (\%) & $5(42)$ & $3(37.5)$ \\
\hline Male gender, no. (\%) & $7(58)$ & $5(62.5)$ \\
\hline Assistant professor & $8(66.6)$ & $5(62.5)$ \\
\hline Associate or full professor & $4(33.3)$ & $3(37.5)$ \\
\hline
\end{tabular}

communicating with patients and families. Conversely, handoffs and transitions of care were perceived to be an area where the open ICU system was beneficial to patient care.

Participants discussed communication challenges that the open ICU placed within the larger interprofessional team. Both hospitalists and intensivists commented on how ICU nurses would frequently be "caught in the middle" or "caught in the crossfire" as a "bystander" or "stepchild."

From a team cohesion [perspective], I really feel for the ICU nurses, they constantly have to make that triage: Do I call ICU? Do I call primary? Most rely on their clinical judgment. When it's a crashing urgent issue, they will pull ICU in because [they are readily available], but page primary. [Hospitalist 8]

It's also challenging for the ICU team because the nurses, the bedside nurses, who are really the unifying force in the patient care, they too have to manage and respond to at least 2 different teams for patient care management. The primary service and the ICU team. Sometimes this works out very smoothly and sometimes it's challenging. [Intensivist 8]

Hospitalists and intensivists frequently cited communication challenges between teams, particularly communication between the ICU and primary teams, referring to "too many cooks in the kitchen" who are "playing a game of telephone."

...What I've seen sometimes, is you have the ICU team presenting, and you have the Medicine [team] presenting the same patient and they're next to each other, which doesn't make a lot of sense to me. [Hospitalist 9]

So there's realizations that when the ICU team is totally out of the loop... We don't even know sometimes when family meetings are. Sometimes we find out about those just in the nick of time. [Intensivist 7]

The presence of consultant teams furthered complexity, leading to individuals being "out of the loop."

Our team doesn't know everything that's happening. This could be... the chest tubes were clamped and nobody 
even knew it, and if the patient coded subsequently, the ICU team wouldn't have even known whether the chest tubes were working because probably it was told to the primary team by IR [Interventional Radiology] but never the ICU team. [Intensivist 9]

Hospitalists and intensivists usually considered transitions of care to be more straightforward; this was perceived as a primary benefit of the open ICU.

Overall the transitions from the ICU to the wards I've found to be pretty seamless here, because of the structure of the ICU, where my team has been following patients for the entire time. This is in juxtaposition to where I trained, in a completely closed ICU, I was able to observe several patients...for very long period of time for very complicated courses would come to the floor and it would be very frequent that there would be issues with those transitions. [Hospitalist 5]

If the primary team has been involved, they're going to know much of what's been in the ICU. They've maintained the relationship with the patient and the family and that is good out on the floor. [Intensivist 7]

However, communication to patients and families was acknowledged as nearly universally "confusing" with high potential for "mixed messages" among teams.

Families... are looking at me and asking me, 'What do you think?' Well, I have to defer to the primary team, and I think they get confused by that. 'But you're my doctor and you're handling the critical part.'...Vents, lines, sedation is talking about machines and objects, and I'm not taking care of machines and objects... I'm taking care of the whole patient. And I'm a pulmonologist and I'm going to talk about their lungs and hearts. [Intensivist 3]

\section{Educational Barriers and Affordances}

Hospitalists and intensivists described barriers and affordances to trainee education in the open ICU system. Hospitalists and intensivists focused on competing demands that learners and educators experienced, particularly other clinical or educational responsibilities.

Learners today are super, super busy...There's a lot of pressure on discharging patients... there's a lot of pressure on moving patients out of the ICU, transitioning patients to lower levels of acuity or tracking multiple volume metrics and patient experience metrics ... They're balancing a lot of competing obligations and interests and priorities. [Hospitalist 1]
Hospitalists and intensivists alike talked of the pressures of balancing service and education:

I think the biggest challenge, not just related to the $I C U$, but to our service in general, is balancing clinical decision-making and the 'work that needs to get done' and teaching...There's a lot of time pressures, the clinical severity and the illness severity of some of the patients, particularly in the ICU, is such that, in order to get through the presentation and the plan, that can take a lot of time. [Hospitalist 6]

I do think that there is a note writing burden that doesn't really actually offer them any opportunity to learn, but we make them do it anyways. [Intensivist 1]

Several participants perceived lack of incentives and institutional support for teaching.

My role in the ICU is a small portion of my overall job... The role of teaching doesn't benefit my career much, so I'm mostly doing it out of kindness of my heart, and when there are competing responsibilities, that seems like one that could fall away. [Intensivist 1]

Many physicians focused on the role of distractions in the busy ICU, such as noise level, interruptions, and pager burden. But the structure of the open ICU also contributed to communication challenges, which also further added distractions.

People have to break off and make a phone call and then wait for a phone call and then once the call comes back, they have to take the phone call. ...This results in...not being able to listen to rounds, and not being able to learn the teaching points. Not being able to participate in the discussion of the next patient probably contributes to not feeling invested in all of the patients. [Intensivist 1]

Hospitalists and intensivists discussed the high medical and educational complexity of ICU patients, particularly the long patient presentations and "compressed" time pressures of rounds, making it feel like there is "never enough time." Both groups acknowledged the challenging role diagnostic uncertainty played in the ICU. Complex procedures, both technical procedures such as intubation and central lines as well as communication or cognitive procedures such as end-of-life conversations, were acknowledged as challenging to teach.

Intensivist faculty more often discussed challenges of teaching teams with members from different professions, specialties, and training levels. 
...Given the [diverse] levels of our learners in our ICU, I find it really challenging...So I often am just focusing on the lowest common denominator, like the medical student and intern level, and really never getting it to those higher-level conversations. [Intensivist 1]

\section{Structural Barriers and Affordances of the Open ICU}

Hospitalists and intensivists also discussed structural barriers and affordances of the open ICU, which impacted both patient care and education. When discussing barriers and affordances of the open ICU, hospitalists were more likely to imply a continuity frame (i.e., benefits of continuity with their patients throughout the hospitalization), whereas intensivists were more likely to cite a fragmentation of care frame (i.e., difficulties dividing care between two different teams). Hospitalists cited great benefits in patient continuity, including "longitudinal relationships" with patients and "smoother transitions" between the ICU and the ward. They also reported that continuity improved education and patient care by helping trainees witness disease evolution, thus serving not only as a structural affordance, but also as an educational affordance.

I watch a trainee misdiagnose a pneumonia in a patient, that ended up being an organizing pneumonia ... Patient developed [acute respiratory distress syndrome], went to the ICU, was intubated, was treated, recovered, and went back to the unit. And the experience of the team managing that patient was like none other, in really appreciating the evolution of disease...the open ICU exposure allows us to see that. [Hospitalist 1]

I really think the continuity of care is very valuable for the teams. For them to be able to start a process and see where it goes as well as the gratification that comes from seeing that an intervention they did is really great in terms of thinking and decision-making. And also the relationships that develop...I think they can be close with patients and families. [Hospitalist 4]

Hospitalists spoke of difficulty balancing ICU and non$I C U$ patients, noting "it is hard to spend the requisite amount of time with an ICU patient when you have 13 other patients that are also pretty sick." [Hospitalist 11] Hospitalists were much more likely to cite geographic delocalization as a related challenge and reported that trainees would often "camp" at bedside in the ICU or spend too long presenting ICU patients, sometimes at the expense of their non-ICU patients.

Hospitalists and intensivists noted "awkwardness" and ambiguity that came with the demarcation of their roles. Intensivists more often reported their teams would cognitively disengage from the patients given the line demarcating their responsibilities, frequently referring to educational "missed opportunities" resulting in "diffusion of responsibility" and lack of true ownership of patients. Some linked cognitive disengagement to feelings of frustration, lower "esprit de corps" (i.e., the feeling of pride and fellowship), and burnout. Many said this exacerbated service-education imbalance, making rounds feel "academic" and not truly patient-care-oriented.

What can really eat at people is the sense of self-worth, the value that they're bringing to patient care, that their presence matters...the prerounding, the presentations, the note-writing...Is anyone going to read my note? [Intensivist 7]

Another common frame where hospitalists and intensivists differed was regarding an expertise versus comanagement frame. Hospitalists more often discussed their lack of ICU expertise and lack of formal ICU curriculum as barriers to education and patient care, leading to feeling "not fully qualified" to teach about certain topics, particularly ventilators. Hospitalists noted wide attending-to-attending "variation" regarding degree of expertise.

The obvious elephant in the room is that we're not board certified in critical care. We may not be as upto-date in the critical care literature. A lot of us go to CME courses and already learn informally from the ICU colleagues... Are we the most appropriate people to be teaching in the ICU? [Hospitalist 8]

Despite this, some hospitalists derived great satisfaction from taking care of critically ill patients, crediting the open ICU system with "bonding" and "personal satisfaction" they derived from relationships with extremely sick patients and families. They invoked the concept of spaced learning, ${ }^{10}$ whereby learning material over time enhances memory compared with learning that occurs all in one session. Participants perceived open ICUs as promoting spaced learning, through which residents could get repeated exposure to ICU patients throughout residency rather than solely through dedicated ICU rotations.

While some hospitalists felt uncomfortable with their lack of ICU expertise, others viewed the open ICU as an explicit advantage: they felt that the availability of ICU team expertise was a "luxury" of the open ICU system as it enabled the patients to get the needed care without "having to worry" about lines and sedation. Some hospitalists similarly felt the open ICU structure provided a "real-world" experience since comanagement is a reality in many community hospitals with open ICU models.

In contrast, intensivists often felt unable to practice medicine at the level they had trained for, often leading to 
"frustration" and job dissatisfaction.

The original reason for creating ICUs was to concentrate skilled personnel from nurses to physicians, now RTs, nutritionists, pharmacists, PTs, in one location where the patient is critically ill. It is hard then to segregate the care when the physicians are not geographically available, as are the nurses. Imagine if the nurses had responsibilities in the ICU and outside the ICU? That would be immediately perceived as nonsensical. Why do we do that for physicians? [Intensivist 8]

\section{DISCUSSION}

Our analysis reveals that open ICUs are challenging environments in which to work that present both affordances and barriers to education of trainees and care for patients. Our findings offer insight into how the educational and patient care experience in open ICUs can be improved for medicine and intensivist teams alike. The discussion will focus on different themes that emerged between hospitalists and intensivists, implications for clinical education and patient care generally, and implications for the structure of workplace learning from comanagement to consultation.

\section{Differential Themes Between Hospitalists and Intensivists}

One of the ways to infer whether hospitalists and intensivists had a "shared mental model" 8 for their work is to examine themes that emerged in discussions with hospitalists versus intensivists. Figure 1 highlights the differences and similarities that hospitalists and intensivists focused on in their analyses of the challenges of education and practicing clinical medicine in the ICU.

Both groups cited similar barriers to clinical care and education in the ICU: competing demands, serviceeducation balance, time pressures, distraction, medical complexity, procedural complexity, and diagnostic uncertainty. Notably, many of these barriers, such as complexity, time pressures, and service-education balance, are not exclusive to the open ICU environment, and both hospitalists and intensivists acknowledged that fact. However, they also acknowledged that these barriers may be magnified by the open ICU structure. Both groups agreed that the structure of the open ICU led to fragmentation of care and role ambiguity and anxiety, where individuals did not understand the division of labor. The psychology and business literature have well-demonstrated that role ambiguity can lead to undesirable outcomes such as decreased satisfaction, decreased organizational effectiveness, and poorer performance. ${ }^{11,}{ }^{12}$ Indeed, several intensivists and hospitalists expressed concerns about exactly these areas.

However, hospitalists' and intensivists' opinions diverged regarding barriers and affordances that were directly related to their respective roles. Hospitalists noted that their lack of ICU expertise, the lack of a formal ICU curriculum, geographic delocalization, and the challenges of balancing ICU and nonICU patients were barriers to teaching effectively. Hospitalists were more likely to cite continuity of care, spaced learning, and job satisfaction as affordances of practicing in an open ICU environment. Specifically, hospitalists noted that continuity not only helped patient care, but also helped learners see the evolution of disease processes and the effects of trainee interventions on the patients.

In contrast, intensivists were more likely to acknowledge team complexity with a diversity of trainees, and learners' cognitive disengagement, as barriers to effective ICU education and patient care. They were more likely than hospitalists to cite concerns about being able to practice at their highest level of training (i.e., scope of practice). While hospitalists reported relying frequently on intensivists' educational content expertise and viewed that access as an affordance of the open ICU, intensivists felt they were not able to fully engage in the ongoing practice necessary to maintain critical care expertise, which promoted dissatisfaction and stagnation. They often felt there was a missed opportunity for true co-construction of the knowledge in the ICU clinical learning environment, which led to perceived loss of professional identity. ${ }^{13}$ Strikingly, intensivists did not identify any structural or educational affordances of the open ICU model (Fig. 1). The lack of professional satisfaction among intensivists suggest that critical care specialists may not thrive within open ICU models, and this could impact trainee education and patient care. Overall, multiple barriers prevented hospitalists and intensivists from truly sharing a mental model when it came to approaching education and patient care in the ICU, despite taking care of the same patients and working with the same learner population.

There has been limited literature on patient outcomes related to ICU structure in the USA, most of which supports benefits for closed ICU systems. Carson et al. ${ }^{14}$ at the University of Chicago studied a cohort of 125 patients before and after their ICU transitioned from an open ICU to a closed model. Their analysis revealed improvement in the metric of "expected mortality" based on illness severity and improvement in ICU RN satisfaction after ICUs were closed. A cohort analysis by Hanson et al. compared outcomes among ICU patient care rendered by dedicated intensivists (i.e., closed system) versus surgeons (i.e., open system). They did not show mortality differences, yet they did find shorter ICU and hospital lengths of stays and lower number of days on mechanical ventilation for the intensivist-only arm. ${ }^{15}$ Treggiari et al. looked at the care of patients with respiratory failure in Seattle ICUs and found that patients in closed ICUs were more likely than those in open ICUs to receive appropriate ventilator settings, and had lower mortality. ${ }^{16}$ While all of these studies suggested benefits of closed versus open ICU structures, none 


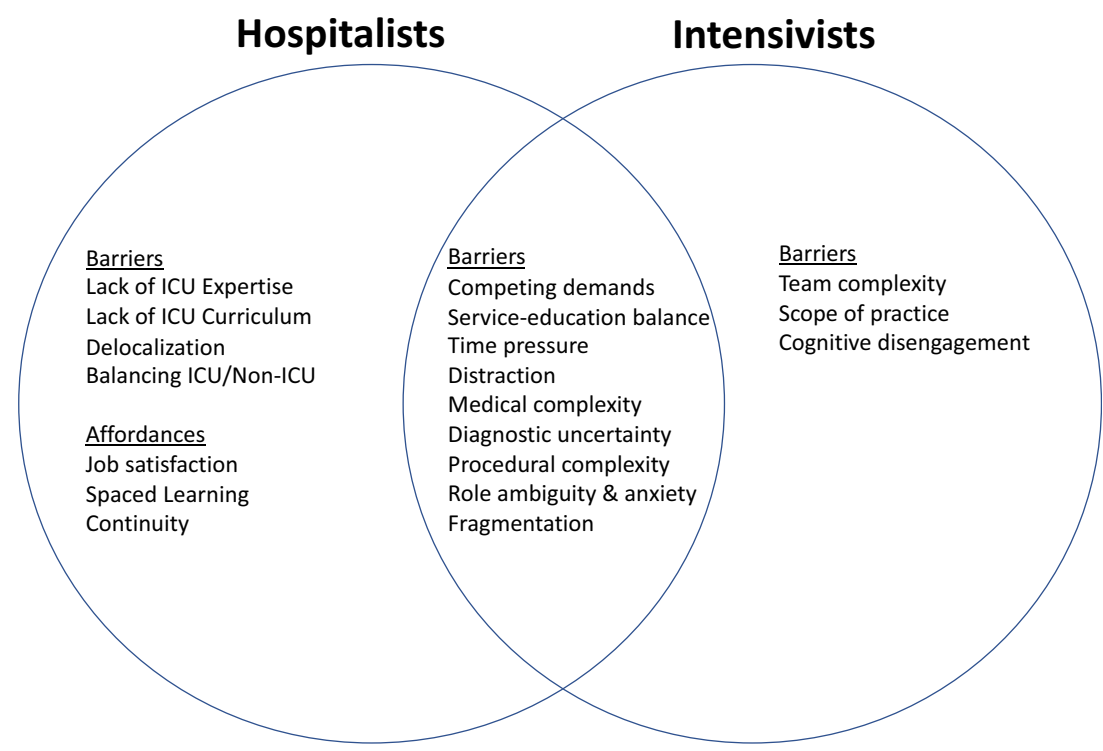

Figure 1 Hospitalists' and intensivists' perceptions of barriers and affordances to education and clinical care in the ICU.

qualitatively assessed in-depth perceptions of practicing clinicians within these ICU structures, and hence, our study adds to the literature.

Barriers and affordances of the open ICU model have implications for the larger concept of medical comanagement. The comanagement model differs from the traditional primary-specialty model. In the latter, consultants make recommendations and the primary team chooses which recommendations to enact or not. In the comanagement model, two or more teams simultaneously share primary responsibility for the care of a patient; in this model, having a shared mental model is crucial to avoid a frenetic approach to patient management. Medical comanagement of surgical patients has been increasing since the rise of the hospitalist movement in the year $2000 .{ }^{17}$ However, training in comanagement may be inadequate, at least from the perspectives of hospitalist physicians in one study. ${ }^{18}$ As complexity of inpatient medical care increases, there remains a crucial need to study and improve comanagement skills, within both medicine and from the interprofessional perspective.

Similarly, the structure of the open ICU also has implications for, and can learn lessons from, the consultative relationship. Consultations are the most common reason for interdisciplinary communication among specialties. ${ }^{19}$ A framework for seven types of consultation in physician communication was recently outlined: ideal, obligatory, procedural, S.O.S., confirmatory, inappropriate, and curbside. ${ }^{20}$ The open ICU model is analogous to a mix of obligatory consultation (i.e., hospital policy mandates consultation for a specific indication) and procedural consultation (i.e., consultation requested to facilitate a procedure). In both of these, friction can be minimized by establishing clear expectations at the beginning of the consultative relationship; otherwise, disappointment and frustration may ensue.
Lessons from comanagement and consultation, and themes from interviews with hospitalists and intensivists, can be instructive in improving education and patient care in the open ICU. Role ambiguity in either a comanagement relationship or an open ICU relationship can be reduced by up-front, frequent, and transparent communication. To mitigate feelings of cognitive disengagement and role anxiety, clear delineation and demarcation of roles and a clear curriculum for all parties would be helpful. To mitigate situations in which one group of physicians feels a lack of expertise whereas another group feels that they are not sufficiently utilizing their expertise, faculty development educational opportunities where both sets of faculty cross-pollinate and develop each other could be beneficial. Acknowledging the shared common patient care and educational barriers that both groups face could also help promote camaraderie as well as improve the learners' experience - all faculty are experiencing the medical complexity and time pressure challenges, and both groups could learn strategies from each other on how to combat these. Learners and interprofessional team members should not be placed in the uncomfortable position of being "caught in the middle" and disagreements should be openly discussed inperson, ideally face-to-face, at the attending level. Similarly, rather than rely on communication between the most junior team members, more routine communication among senior residents and attending physicians should be formalized. Barriers to communication between teams should be removed and efforts to establish joint in-person rounding should be attempted as much as possible. In the absence of joint inperson rounding, a dedicated time period should be set aside every day for communication about these comanaged patients. Box 3 summarizes the barriers to patient care and education and proposes improvement strategies to address these challenges. 
Box 3 Barriers to education and patient care and proposed improvement strategies

\begin{tabular}{|c|c|}
\hline Barriers & Proposed improvement strategy \\
\hline Role ambiguity & $\begin{array}{l}\text { Up-front, frequent, and transparent } \\
\text { communication }\end{array}$ \\
\hline Cognitive disengagement & $\begin{array}{l}\text { Clear delineation and demarcation of } \\
\text { roles, clear curriculum for all parties }\end{array}$ \\
\hline Lack of ICU expertise & $\begin{array}{l}\text { Creation of faculty development } \\
\text { educational opportunities to cross- } \\
\text { pollinate and develop each other }\end{array}$ \\
\hline $\begin{array}{l}\text { Shared barriers-medical } \\
\text { complexity, time pressure } \\
\text { challenges, etc. }\end{array}$ & $\begin{array}{l}\text { Faculty acknowledgment of share } \\
\text { common patient care and educational } \\
\text { barriers }\end{array}$ \\
\hline Communication challenges & $\begin{array}{l}\text { Avoid placing learners or } \\
\text { interprofessional team members "in } \\
\text { the middle"-disagreements should } \\
\text { be openly discussed in-person, } \\
\text { ideally face-to-face, at the attending } \\
\text { level }\end{array}$ \\
\hline Communication challenges & $\begin{array}{l}\text { Formalize more communication } \\
\text { between the teams at a senior } \\
\text { resident/attending level and efforts } \\
\text { should be made to establish joint in- } \\
\text { person rounding, or at least a } \\
\text { dedicated time period to discuss } \\
\text { comanaged patients }\end{array}$ \\
\hline
\end{tabular}

\section{Study Limitations}

Our findings should be interpreted within the context of our methodological choices. Although this was a single-centered study, which limits generalizability of our findings, it is the only known qualitative study exploring perceptions of open versus closed ICU models from the perspectives of intensivist and hospitalist faculty. Thus, this study can serve as a pilot study to inform larger qualitative explorations of discussions around ICU structures, staffing, and educational models. Because we aimed to better understand lived experiences of teaching in open ICUs, we did not recruit from closed ICU settings. We therefore cannot compare open to closed ICU experiences; this was not our goal but could be illuminating to study in the future. We interviewed only teachers in order to enrich both content and pedagogical knowledge, but a future learner-centered study would be helpful to explore learners' perspectives on learning in the open ICU environment. Likewise, because we sought to deeply examine the experience of physicians in the open ICU, we did not interview subjects from other professions, such as nurses or techs.

\section{CONCLUSIONS}

In summary, this qualitative study elucidated perceptions of hospitalists and intensivists clinically practicing and educating in an academic open ICU structure. We found that hospitalists and intensivists shared mental models for barriers to patient care and education in the open ICU, but had divergent perceptions of affordances of the open ICU system. Our findings reveal practical suggestions for how hospitalists and intensivists can bridge divides and concretely adopt strategies to improve patient care and education in this unique staffing structure. This study not only provides insight into the general challenges of education, learning, and patient care in the open ICU, but can also inform larger qualitative explorations of discussions around ICU structures, staffing, and educational models.

Acknowledgments: The authors thank the study participants for their time spent in the semi-structured interviews.

Corresponding Author: Lekshmi Santhosh, M.D., M.A.Ed.; Division of Hospital Medicine University of California-San Francisco, S1183, 505 Parnassus Avenue, San Francisco, CA 94143, USA (e-mail: Lekshmi.Santhosh@ucsf.edu).

Funding Information This project was funded by the UCSF Department of Medicine and the UCSF Nina Ireland for Lung Health Foundation.

\section{Compliance with Ethical Standards:}

Conflict of Interest: There are no conflicts to disclose.

\section{REFERENCES}

1. Gutsche JT, Raiten JM. Staffing models for the ICU: open, closed, MD, NP, or telemedicine? Curr Anesthesiol Rep 2013;3(2):65-72.

2. Gesenway, D. The tug-of-war over ICU care. Today's Hospitalist. 2009. Available at: https://www.todayshospitalist.com/the-tug-of-war-overicu-care/.

3. Minter, D. J., Levy, S. D., Rao, S. R., \& Currier, P. F. (2018). Intensive care unit rotations and predictors of career choice in pulmonary/critical care medicine: a survey of internal medicine residency directors. Critical Care Research and Practice, 2018.

4. Santhosh L, Brown W, Ferreira J, Niroula A, Carlos WG. Practical tips for ICU bedside teaching. Chest. 2018;154(4):760-765.

5. Brzezinski M, Kukreja J, Mitchell JD. Time-efficient, goal-directed, and evidence-based teaching in the ICU. Curr Opin Anaesthesiol. 2019.

6. Joyce MF, Berg S, Bittner EA. Practical strategies for increasing efficiency and effectiveness in critical care education. World $J$ Crit Care Med. 2017;6(1):1-12. Published 2017 Feb 4. https://doi.org/10.5492/ wjccm.v6.i1.1

7. Malterud K, Siersma VD, Guassora AD. Sample size in qualitative interview studies: guided by information power. Qualit Health Res. 2015.

8. Nakarada-Kordic I, Weller JM, Webster CS, et al. Assessing the similarity of mental models of operating room team members and implications for patient safety: a prospective, replicated study. BMC Med Educ 2016;16(1):229.

9. Brooks J, McCluskey S, Turley E, King N. The utility of template analysis in qualitative psychology research. Qual Res Psychol 2015;12:202-222.

10. Ebbinghaus, H. (1913). Memory, Trans., Trans. H. A. Ruger and C. E. Bussenius. New York: Teachers College.

11. Ebbers JJ, Wijnberg NM. Betwixt and between: role conflict, role ambiguity and role definition in project-based dual-leadership structures. Hum Relat 2017;70(11):1342-1365. https://doi.org/10.1177/ 0018726717692852.

12. Jackson SE, Schuler RS. A meta-analysis and conceptual critique of research on role ambiguity and role conflict in work settings. Organ Behav Hum Decis Process 1985;36(1): 16-78.

13. Gutiérrez KD, Baquedano-López $\mathbf{P}$, Tejeda C. Rethinking diversity: hybridity and hybrid language practices in the third space. Mind Cult Act 1999;6(4):286-303.

14. Carson, S. S., Stocking, C., Podsadecki, T., et al. (1996). Effects of organizational change in the medical intensive care unit of a teaching hospital: a comparison of 'open' and 'closed' formats. JAMA, 276(4), 322328.

15. Hanson CW 3rd, Deutschman CS, Anderson HL 3rd, Reilly PM, Behringer EC, Schwab CW, et al. Effects of an organized critical care 
service on outcomes and resource utilization: a cohort study. Crit Care Med 1999;27(2):270-4.

16. Treggiari MM, Martin DP, Yanez ND, Caldwell E, Hudson LD, Rubenfeld GD: Effect of intensive care unit organizational model and structure on outcomes in patients with acute lung injury. Am J Respir Crit Care Med 2007, 176: 685-690. https://doi.org/10.1164/rccm. 200701-165OC.

17. Sharma G, Kuo Y, Freeman J, Zhang DD, Goodwin JS. Comanagement of hospitalized surgical patients by medicine physicians in the United States. Arch Intern Med 2010;170(4):363-368. https://doi.org/10.1001/ archinternmed.2009.553
18. Plauth WH III, Pantilat SZ, Wachter RM, Fenton CL. Hospitalists' perceptions of their residency training needs: results of a national survey. Am J Med 2001;111 (3) 247- 254.

19. Conley J, Jordan M, Ghali WA. Audit of the consultation process on general internal medicine services. Qual Saf Health Care 2009;18:59-62.

20. Hale AJ, Freed JA, Alston WK, Ricotta DN. What are we really talking about? An organizing framework for types of consultation and their implications for physician communication. Acad Med. 2019;94(6):809-812.

Publisher's Note: Springer Nature remains neutral with regard to jurisdictional claims in published maps and institutional affiliations. 